\author{
Rafael Stryjer \\ Daniel Grupper \\ Rael Strous \\ Centro de Salud Mental Beer Yaakov, \\ Beer Yaakov 70350, Israel \\ Facultad Sackler de Medicina, Universidad de Tel Aviv, \\ Tel Aviv, Israel \\ Michael Poyurovsky \\ Centro de Salud Mental Tirat Camel, \\ Tirat Carmel, Israel \\ Facultad de Medicina, \\ Instituto de Israel de Tecnología (Technion), \\ Haifa, Israel \\ Abraham Weizman \\ Facultad Sackler de Medicina, Universidad de Tel Aviv, \\ Tel Aviv, Israel \\ Centro de Salud Mental Geha y Centro reinvestigación \\ Médica Felsenstein, \\ Campus Beilinson Petah Tiqva, Israel
}

\section{Administración nasogástrica de risperidona para tratar las ideas delirantes de envenenamiento}

Querido señor:

M. tenía 15 años cuando lo derivaron al Servicio del Niño y del Adolescente en un estado que se pensaba amenazaba su vida. Este caso es interesante por su administración de la medicación antipsicótica y porque hay datos sugestivos de que tanto el abuso de cannabis [1] como la pérdida rápida de peso [2] se asocian con trastornos mentales.

M. había sido usuario regular de cannabis desde hacía 2 años. Su conducta cambió hacía un año, cuando el paciente se convirtió en una persona retirada socialmente. Dejó de ir a la escuela, creyendo que sus compañeros se reían de él y porque tenía dificultad para concentrarse. Mencionó el suicidio y no se ocupaba de sí mismo. Dormía poco y se tendía en el suelo, gritando y llorando con voz extraña. M. había perdido peso; no había comido casi nada durante las tres últimas semanas y, temeroso de que lo envenenaran, sólo tomaba un trago de agua si su hermano bebía primero del vaso.

M. se había trasladado a Inglaterra a los 10 años de edad y se estableció sin problemas importantes. Sus padres se habían divorciado hace 9 años. Su madre sufría depresión; su padre había experimentado antes un episodio psicótico y depresión y había sido adicto al cannabis y la heroína, y su hermano había utilizado cannabis.

Cuando acudió a la consulta, M. estaba descuidado, pálido y delgado; la debilidad dificultaba la marcha. Hablaba unas pocas palabras en un susurro y había datos sugestivos de interferencia del pensamiento, pues encontraba difícil hablar y no podía elaborar. Se diagnosticó a M. psicosis no orgánica no especificada (CIE-10; F29).
Debido a su fragilidad, se ingresó a M. en una sala médica general para rehidratación inmediata. Se prescribió 2,5 $\mathrm{mg}$ de olanzapina, que aumentaron a $5 \mathrm{mg}$ al día siguiente, pero el paciente se negó a tragar las tabletas y se le dio una inyección de $5 \mathrm{mg}$ de haloperidol. El día siguiente, M. mostraba rigidez grave. Se interrumpió el haloperidol y se iniciaron $5 \mathrm{mg}$ diarios de olanzapina (Velotab $\mathbb{R}$ ) y $50 \mathrm{mg}$ diarios de sertralina. El incumplimiento de la medicación y la psicosis persistía de manera que, cuando se inició la alimentación nasogástrica en su quinto día de estancia en el hospital, se interrumpió toda la medicación y se administró $1,5 \mathrm{mg}$ diarios de risperidona líquida vía sonda nasogástrica.

La mejoría fue rápida. Durante el par de días siguiente comenzó a comer, beber, hablar y caminar. Fue transferido a una sala psiquiátrica y enviado a casa con el alta con 2 $m g$ diarios de risperidona líquida después de 2 semanas. El paciente se mantuvo estable hasta que utilizó cannabis bebiendo cerveza. Esto causó cierto deterioro en el estado mental, de manera que se aumentó la risperidona a $3 \mathrm{mg}$ diarios y se advirtió al paciente contra el uso de cannabis. Transcurridos 6 meses, M. está estable; come y bebe normalmente, atiende a su higiene personal y participa en actividades familiares.

Hay datos que indican una asociación entre la psicosis y el abuso de cannabis [3]. En efecto, cada vez hay más datos de que el sistema cannabinoide puede estar implicado en los trastornos psicóticos $[4,5]$. Nuestro paciente apoya a otros [1] en que el uso de cannabis (i) precipita trastornos psicóticos en individuos vulnerables (es decir, pacientes con riesgo alto, p. ej., historia familiar) y (ii) exacerba los síntomas de psicosis en quienes continúan utilizando cannabis.

Creemos que éste es el primer informe de administración de risperidona a través de una sonda nasogástrica. Era apropiado en este paciente que se negaba a tragar por temor a ser envenenado. Se ha asociado también la perturbación psiquiátrica con pérdida de peso considerable y rápida mediante la dieta, y la vuelta a la dieta y el peso normales se ha traducido en una mejoría del trastorno [2]. Aunque cierta mejoría se puede haber debido a la alimentación y la ganancia de peso consiguiente, se consideró que el tratamiento de risperidona fue lo que desempeñó un papel importante en la rehabilitación de este paciente.

\section{Bibliografía}

[1] Degenhardt L, Hall W. Cannabis and psychosis. Curr Psychiatry Rep 2002;4:191-6.

[2] Glass M. The role of cannabinoids in neurodegenerative diseases. Prog Neuropsychopharmacol Biol Psychiatry 2001;25:743-65.

[3] Hambrecht $M$, Häfner $\mathbf{H}$. Cannabis, vulnerability, and the onset of schizophrenia: an epidemiological perspective. Aust NZ J Psychiatry 2000;34:468-75.

[4] Robinson S, Winnik HZ. Severe psychotic disturbances following crasch diet weight loss. Arch Gen Psychiatry 1973;29:559-62. 
[5] Skosnik PD, Sparz-Glenn L, Park S. Cannabis use is associated with schizotypy and attentional dysinhibition. Schizophrenia Res 2001;25:743-65.

\section{Jawaid A. Khan \\ Beryl Langley \\ Hospital Edith Cavell, Breton Gate, Peterborough PE3 6GZ, Reino Unido}

\section{Escitalopram en la tricotilomanía}

\section{Señor:}

La tricotilomania es un trastorno psiquiátrico olvidado con expresión dermatológica [1]. A pesar de nueva información intrigante sobre la tricotilomanía, se carece de la información básica sobre su prevalencia, evolución natural y tratamiento [2]. La tricotilomanía es un trastorno de los impulsos en el que los pacientes se tiran crónicamente del pelo del cuero cabelludo o de otros lugares, o ambas cosas [3]. Tirarse del cabello y arrancárselo es más común en las regiones frontoparietales y temporales de la cabeza, aunque ocasionalmente pueden estar implicadas las pestañas, las cejas, el vello púbico y el vello en otros sitios del cuerpo. Informamos sobre un niño que presentaba pérdida gradual del pelo en el cuero cabelludo y había también compulsión a arrancárselo a otros miembros de la familia. Respondió a un nuevo ISRS, el escitalopram.

S., un niño de 10 años que estudia $5^{\circ}$ curso de la enseñanza reglada, fue traído por su padre a la clínica dermatológica por pérdida de pelo en la cabeza durante los 10 últimos meses. El niño era normal unos 10 meses atrás, cuando desarrolló un área de alopecia en el cuero cabelludo. Se prescribieron al niño medicamentos antimicóticos junto con una loción para el cuero cabelludo durante 2 meses sin ninguna mejoría. El hábito de arrancarse el cabello había aumentado y también comenzó a arrancárselo a su madre y hermana siempre que tenía la oportunidad, especialmente cuando estaban dormidas. El examen del pelo del cuero cabelludo del niño era normal. Se derivó al niño para evaluación psicológica. Se interrumpieron todas las medicaciones. En una evaluación psicológica detallada en tres sesiones, se encontró que no se permitía al niño salir fuera a jugar con los amigos y estaba confinado únicamente a la casa. Esto se debía al miedo de los padres a que el niño pudiera malcriarse o sufrir un accidente. No se permitía al niño ninguna actividad de ocio en casa y le pedían continuamente que estudiara o durmiera. El niño tenía también el hábito de morderse las uñas. Era el más pequeño de tres hermanos. No había antecedentes propios o familiares de ninguna enfermedad física o psiquiátrica crónica. El padre tenía una tienda de muebles en la propia casa. El niño tenía un buen expediente académico. Después de desarrollar este problema, el niño llevaba gorra todo el tiempo, incluso durante el verano. Después de desarrollar la enfermedad, comenzó a recibir atención de los miembros de su familia.

Se iniciaron $5 \mathrm{mg}$ diarios de escitalopram con el niño, que se aumentaron a $10 \mathrm{mg}$ diarios después de una semana, y se dio también sugestión, distracción y explicación de la naturaleza de los sintomas. Se aconsejó también al padre sobre la naturaleza de la enfermedad, su comienzo y perpetuación. Hubo una disminución gradual en la frecuencia del arrancamiento del cabello y de morderse las uñas, seguido por remisión completa. En el seguimiento hasta los 2 meses, no había desarrollado el hábito y comenzaba a disfrutar de actividades en el exterior con sus amigos.

La tricotilomanía en niños se asocia comúnmente con otros rasgos neuróticos como morderse las uñas (como se asociaba en el presente caso), chuparse el dedo, ansiedad o estrés por los exámenes, discapacidad de aprendizaje y negligencia de los padres [4], y puede haber ingestión de pelo que lleve a tricobezoar. En el presente caso, había factores causantes de estrés, en forma de negligencia y aburrimiento. Se ha advertido un aumento de la incidencia de trastorno obsesivo-compulsivo (TOC) comórbido con la tricotilomanía y las investigaciones neurológicas han corrido paralelas con estudios etiológicos del TOC y han demostrado tanto similitudes como diferencias entre el TOC y la tricotilomanía [5]. Algunos investigadores han etiquetado incluso la tricotilomanía como un trastorno del espectro del TOC. Aunque se ha encontrado que los inhibidores selectivos de la recaptación de serotonina, especialmente la fluoxetina, la paroxetina, la fluvoxamina y el citalopram son útiles en la tricotilomanía [6-10], no hay informes sobre el papel del escitalopram (que es un ISRS sumamente selectivo y S-enantiómero puro del derivado ftalano bicíclico racémico citalopram) en la tricotilomanía. Es preciso reconocer a tiempo y tratar apropiadamente el trastorno para evitar cualquier complicación futura.

\section{Bibliografía}

[1] Bhatia MS, Singhal PK, Rastohi V, Dhar NK, Nigam VR, Taneja SB. Clinical profile of trichotillomania. J Indian Med Assoc 1991;89:137-8.

[2] Block C, West SA, Baharoglu R. Paroxetine treatment of trichotillomania in an adolescent. J Child Adolesc Psychopharmacol 1998;8:69-71.

[3] Bhatia MS. Trichotillomania: an obsessive compulsive spectrum disorder. Indian J Dermatol 1995;40:134-5.

[4] Christense GA, Crow SJ. The characterization and treatment of trichotillomania. J Clin Psychiatry 1996;57(Suppl 8):42-7.

[5] Hautman G, Hercogova J, Lotti T. Trichotillomania. J Am Acad Dermatol 2002;46:807-21.

[6] Jaspers JP. The diagnosis and psychopharmacological treatment of trichotillomania: a review. Pharmacopsychiatry 1996;29:115-20.

[7] Stanley MA, Breckenridge JK, Swann AC, Freeman EB, Reich L. Fluvoxamine treatment of trichotillomania. J Clin Psychopharmacol 1997;17:278-83.

[8] Stein DJ, Bouwer C, Maud CM. Use of the selective serotonin reuptake inhibitor citalopram in treatment of trichotillomania. Eur Arch Clin Neurosci 1997;247:234-6.

M. S. Bhatia UCMS, D-1 Naraina Vihar, Delhi 110028, India.

Savita Sapra AIIMS, Nueva Delhi 110029, India 